\title{
Detoxification and anti-mycotoxigenic effect of 0chratoxin A produced by Penicillium expansum species utilizing probiotic Pichia kudriavzevii and biogenic-selenium nano-formulations
}

\author{
Eman Ahmed Mohamed Helmy* \\ The Regional Center for Mycology and Biotechnology (RCMB), Al-Azhar University, Cairo, Egypt.
}

\begin{tabular}{l}
\hline ARTICLE INFO \\
\hline Received on: 13/04/2018 \\
Accepted on: 06/07/2018 \\
Available online: 07/03/2019 \\
\\
\hline Key words: \\
OTA, Detoxification, \\
Probiotic, Pichia kudriavzevii, \\
Biogenic-Selenium Nano.
\end{tabular}

\section{INTRODUCTION}

Egypt is predominately an agricultural nation with almost all its kin relying upon farming or provincial economy. It is obvious that with the low yield efficiency, the anticipation of pre- and post-harvest losses of horticultural items is extremely imperative for beating the developing nourishment request. Preparing of nourishment grains for wellbeing and nature of prepared items is yet a challenge for the food industry. Fungi represent a critical risk to nourishment wellbeing, of which mycotoxin sullying is

\footnotetext{
*Corresponding Author

Eman Ahmed Mohamed Helmy, , The Regional Center for Mycology and

Biotechnology (RCMB), Al-Azhar University, Cairo, Egypt.

E-mail: emanhelmo@yahoo.com
}

This article was presented at the 5th Euro-Mediterranean Conference and Expo on Life Sciences, Pharma and Biomedicine (BioNat-V), Limassol, Cyprus. one of the principle concerns. As of late poultry and domesticated animals, the feed industry has picked up noticeable quality in the national economy, Gabriel and Puleng (2013).

Among the mycotoxigenic microorganisms introduced in poultry encourages, Penicillium species and their mycotoxins are accounted for to influence encourage quality and human health. Mycotoxins cause a wide scope of creature medical issues, for example, diminishment in creature profitability, immunosuppression, harm to essential organs, barrenness, and in outrageous cases, demise may happen (Fink-Gremmels and Malekinejad, 2007). Ochratoxin A (OTA) is a standout amongst the most critical mycotoxins of overall worry for human and creature wellbeing. It shows an extensive variety of wellbeing impacts including nephrotoxicity, mutagenicity, teratogenicity, and immunotoxicity (O'Brien and Dietrich, 2005), influences protein union and restrains Adenosine Tri-Phosphate (ATP) creation 
(Koteswara et al., 2016). Molds decay food and feed causes extraordinary monetary misfortunes around the world. It is assessed that near $5 \%$ and $10 \%$ of the world's food creation is squandered because of contagious deterioration (Khalil et al., 2013).

Studies did in a few nations including Tunisia, Egypt, and France have shown a connection between dietary admission of OTA and the improvement of renal and urothelial tumors. OTA is known to influence the insusceptible framework in various mammalian species. OTA causes immunosuppression following pre-birth, postnatal, and grown-up life exposures. These impacts incorporate lessened phagocytosis and lymphocyte markers and expanded defenselessness to bacterial contaminations and postponed reaction to vaccination in piglets. OTA initiates apoptosis in an assortment of cell writes in vivo and in vitro that interceded through cell forms associated with the corruption of DNA. Besides, the immunosuppressant action of OTA is described by estimate lessening of crucial safe organs, for example, thymus, spleen, and lymph hubs, sorrow of neutralizer reactions, modifications in the number and elements of safe cells, and tweak of cytokine generation (Tumor Necrosis Factor- $\alpha$ (TNF- $\alpha$ ) and interleukin-6 (IL-6)), Gabriel and Puleng (2013).

A few procedures (physical and chemical) have been created to avoid and additionally to diminish mycotoxin collection in grains. These are costly and not commonsense to actualize on farming fields (Dalie et al., 2012). In the meantime, a few scientists have concentrated on detoxification of mycotoxins by biotransformation responses which incorporate acetylation, hydrolysis, deamination, and decarboxylation (McCormick, 2013). A promising system to lessen mycotoxin pollution in nourishments and encourages is to include the biotic cooperation between the nontoxigenic and toxigenic strains of similar species (Dorner et al., 2003). However, there are no such examinations because of indigenous mycobiota on OTA generation in poultry nourish by Penicillium species (Koteswara et al., 2016).

The use of gamma radiation is another technique used for preserving food that may exert some effects on mycotoxins. In a recent study by Thalita et al. (2018), OTA was irradiated in its dry form, in aqueous and in methanolic solutions, and in wheat flour, grape juice, and wine. In aqueous solutions, more than $90 \%$ of the OTA was degraded by $\gamma$-radiation doses $\geq 2.5 \mathrm{kGy}$, and a two-fold reduction in OTA cytotoxicity was observed. In food matrices, the elimination of OTA by $\gamma$-radiation was found more difficult, as radiation doses of $30 \mathrm{kGy}$ eliminate at most $24 \%$ of the OTA. Higher moisture content of food matrices did not substantially increase OTA elimination because OTA is very sensitive to irradiation in water solutions but resistant in its dry form and in food matrices.

In fact, mycotoxins have been appeared to be the main risk among nourishment and bolster contaminants with respect to incessant poisonous quality. The difficulties introduced to those working in mycotoxin investigate are huge because of the recurrence, the many-sided quality, and inconstancy in the event. A few viewpoints by authors including Saeger et al. (2016) make the pre- and post-reap control of mycotoxins troublesome, for example, different parasitic species deliver mycotoxins; most of the mycotoxins-creating organisms can produce more than one mycotoxin; mycotoxins levels are affected by natural conditions amid development and capacity; the nearness of altered mycotoxins; the exceptionally complex impact of ecological factors on the biosynthesis of mycotoxins by growths.

Different angles identified with human and creature wellbeing additionally add to the multifaceted nature in mycotoxin examine, e.g., the absence of appropriate biomarkers to survey presentation of people and creatures; the requirement for direction levels of mycotoxins in creature body liquids; the viability and security testing of mycotoxin detoxifiers; and knowledge about toxicokinetic in creatures. New improvements in mycotoxin examination concentrate on speedier, multi-mycotoxin, earth amicable, financially savvy, and fit-for-reason techniques in nourishment, sustain organic tissue and body liquids, Aniket et al. (2018).

Mycotoxigenic organisms, mycotoxins and sustenance and bolster security will keep on being a basic enthusiasm to specialists for quite a long time to come. Advancements happen at a fast pace. In any case, only through multidisciplinary endeavors and purposeful activities can additionally advance and arrangements are normal for the mycotoxins issue (Saeger et al., 2016).

Concerning the probiotic yeasts, Leonardo et al. (2016) tested the ability of some probiotic yeasts to remove OTA in the gut. According to their outcomes, S. cerevisiae W13 and $S$. cerevisiae var. boulardii ATCC MYA-796 were found as the most promising strains due to their ability to remove $21 \%$ of OTA.

Another study by Khalil et al. (2013) screened five strains of lactic acid bacteria (LAB) for their capacity to repress Fusarium species development and additionally tie its secreted mycotoxin; fumonisin B1. The promising results indicated that $L b$. delbrueckii subsp. lactis DSM 20076 was the most productive endure expelling fumonisin B1 by $76.67 \%$. However, Lb. acidophilus DSM 20079 , Lb. sakei LB 706, and Pediococcus acidilactici NNRL B-5627 demonstrated the best inhibitory impact against tested Fusarium strains. Subsequently, the utilization of LAB as a wellspring of normal antimycotic and antimycotoxin specialists demonstrated a promising, prudent, and effective technique for protecting nourishment items from Fusarium deterioration.

Recently, different strategies have been used for the reduction of the mycotoxins from the contaminated feed to protect animals against the harmful effects of them. Various natural and synthetic agents are known to prevent both mycotoxigenic mold's growth and mycotoxin formation (Mahoney et al., 2010). Not long ago, nanoparticle (NP) materials have received increasing attention due to their unique physical and chemical properties, which differ significantly from their conventional counterparts (Stoimenov et al., 2002).

Among the allowed traditional huge forms of nanomaterials as food additives by European Food Safety Authority are $\mathrm{SiO}_{2}, \mathrm{E} 551$, and $\mathrm{TIO}_{2}, \mathrm{E} 171$. Nano silica is known to be used in food contact surfaces, food packaging, in dried soups as they are free-flowing and in the clearing of beer and wine. Second Nano Titanium is used in a number of products including paints, coatings, and food oxide. Recently, nano-selenium is used as an additive in tea output and it is currently using functionalized NPs that contain certain functions of the surface and added mainly in food packaging applications (Helmy, 2016).

Accordingly, Asmathunisha and Kathiresan (2013) reported that the synthesis of NPs may be triggered by several compounds such as carbonyl groups, terpenoids, phenolics, 
Table 1. Strains of Penicillium expansum preserved at RCMB, Al-Azhar University, Egypt.

\begin{tabular}{cc}
\hline Isolate no. & Accession number \\
\hline 1. & IMI 28169 \\
2. & RCMB $001001(1)$ \\
3. & RCMB $001001(2)$ \\
4. & RCMB $001001(3)$ \\
5. & RCMB $001001(4)$ \\
6. & RCMB $001001(5)$ \\
7. & RCMB $001001(6)$ \\
\hline
\end{tabular}

flavones, amines, amides, proteins, pigments, alkaloids, and other reducing agents presenting in the plant extracts and microbial cells. Fungal cell free-water extract is an environmentally agreeable reducing agent for metal-nano-materials formation. The inhibitory effect of metal NPs on the growth and production of mycotoxins by mycotoxigenic fungi was previously studied by Yehia and Ahmed (2013). They recorded that Zinc oxide (ZnO) NPs control the production of the mycotoxins fusaric acid and patulin produced by Fusarium oxysporum and Penicillium expansum, respectively, in a concentration-dependent manner. Similarly, El-Desouky and Ammar (2016) indicated that $3 \mathrm{mg} / 100 \mathrm{ml}$ media of honey derived Silver nanoparticles (AgNPs) have reduced the aflatoxins G1, G2, B1, and B2 production by Aspergillus parasiticus to $77.55 \%, 62.91 \%, 58.76 \%$, and $66.56 \%$, respectively and OTA by Aspergillus ochraceous to $79.85 \%$ with significantly inhibitory effect on mycelial growth.

The effects of ozone treatment on zearalenone (ZEN) and OTA and quality of ozonized corn were recently investigated by Lijun et al. (2016). Ozone significantly affects ZEN and OTA solutions. ZEN was undetectable 5 seconds after being treated with $10 \mathrm{mg} \mathrm{l}^{-1}$ ozone. However, OTA was resistant to ozonation with a diminution rate of $65.4 \%$ after 120 seconds of treatment. Moreover, ZEN and OTA solutions were difficult to degrade after being dried by a nitrogen stream. Their results showed that ozone effectively degraded ZEN and OTA in corn. The diminution rates of ZEN and OTA in corn increased with ozone concentration and treatment time.

There were no previous studies reporting the effect of mycogenic-Selenium nanomaterials (SeNPs) nor probiotic yeast Pichia kudriavzevii on the fungal production of OTA neither of the fungal ultrastructure tolerance to such formulation. Therefore, the aim of this study research was to evaluate the combined inhibitory capacity of SeNPs and probiotic yeast on OTA production by $P$. expansum species as well as their ultrastructure acclimatization.

\section{MATERIALS AND METHODS}

\section{Fungal strains}

Seven fungal strains of Penicillium expansum species (Fig. 1B and Table 1) were examined for their capabilities to produce OTA Figure 1A using thin-layer chromatography (TLC) (Fig. 2). They were kindly provided by culture collection unit (CCU) of the Regional Centre for Mycology and Biotechnology (RCMB), Al-Azhar University, Cairo, Egypt. The probiotic yeast species, Pichia kudriavzevii, were earlier isolated from Egyptian buffalo sweetened Karish cheese, identified (morphologically, biochemically, and genotypically) as well as proved for their probiotic characteristics by Helmy and Abdul Aziz (2018).

\section{Chemicals and analytical standards}

Standard OTA (Fig. 1A) was purchased from Sigma (St. Louis, MO). Stock solution of standard OTA was prepared and assayed according to Association of Official Analytical Chemists (AOAC) (2005) in acetonitrile/methanol concentration of $[1: 1$ $(\mathrm{v} / \mathrm{v})]$, stored in an amber vial in a freezer $\left(\mathrm{ca} .-18^{\circ} \mathrm{C}\right)$. All solvents were of high-performance liquid chromatography (HPLC) grade. All chemicals and solvents were obtained from Sigma-Aldrich, France. The water was double distilled with Millipore water purification system (Bedford, MA).

\section{Biogenic selenium nanoparticles}

Se-nanoparticles (SeNPs) were beforehand accomplished by Helmy et al. (2018). They benefited of using fungal cell-free water extract of Trichoderma harzianum containing the exact reducing enzyme Nicotinamide Adenine Dinucleotide Phosphate (NADPH)-dependent dehydrogenase vital for the reduction of $10 \mathrm{mM}$ of the inorganic meta salt of sodium selenite $\left(\mathrm{Na}_{2} \mathrm{SeO}_{3}\right)$ to selenium nanospheres of $16 \mathrm{~nm}$ in diameter. Se-nanoparticles (SeNPs) were prepared as a single watery treatment and with probiotic Pichia kudriavzevii in a concentration of $[1: 1(\mathrm{v} / \mathrm{v})]$ and as consolidated plans of both to look at their capability to dispose of, detoxificate, and additionally lessening of this mycotoxin.
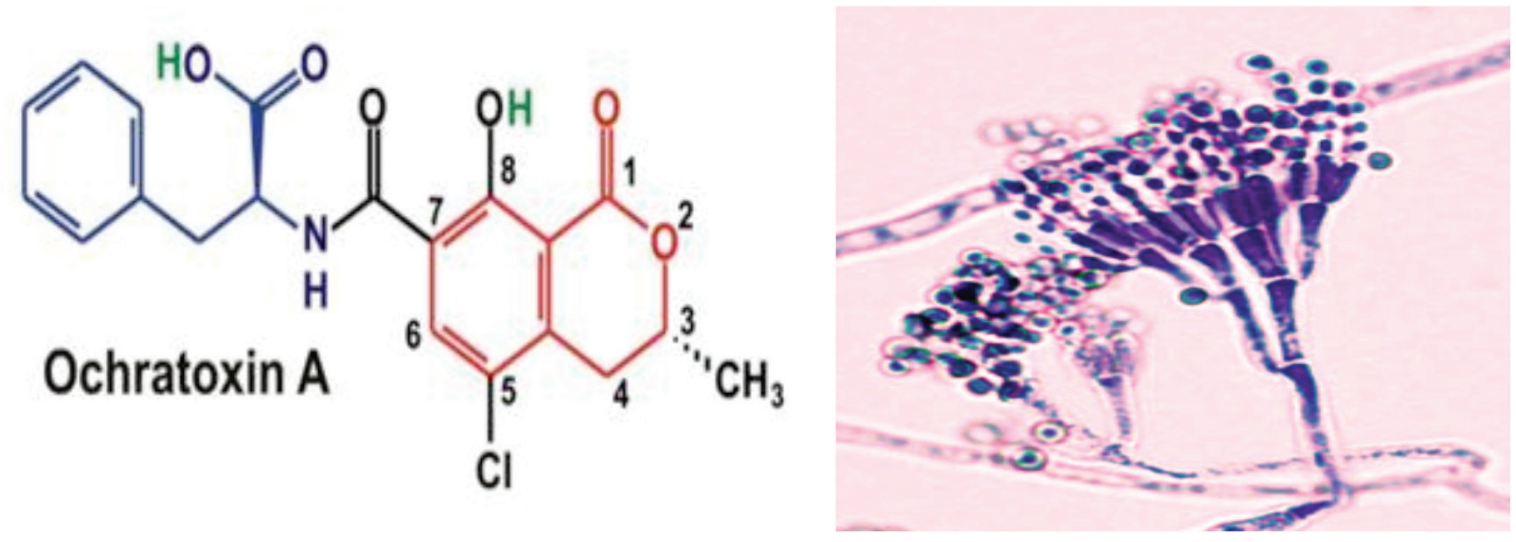

Figure 1. (A) OTA Chemical Structure and (B) Penicillium expansum Morphological Characters, $40 \times$. 
Table 2. Quantitative determination of OTA productivity (\%) produced by $P$. expansum strains treated with P. kudriavzevii and SeNPs formulation using HPLC.

\begin{tabular}{|c|c|c|c|c|c|c|c|}
\hline \multirow[t]{2}{*}{ Treatments } & \multicolumn{7}{|c|}{ OTA productivity (\%) } \\
\hline & P. $\exp 1$ & Р. $\exp 2$ & Р. $\exp 3$ & Р. exp 4 & Р. $\exp 5$ & Р. $\exp 6$ & P. $\exp 7$ \\
\hline Control* & 70.47 & 76.48 & 69.91 & 72.67 & 73.08 & 72.41 & 70.7 \\
\hline SeNPs & 62.62 & 65.53 & 62.97 & 63.61 & 62.01 & 63.22 & 63.15 \\
\hline P. kudriavzevii & 51.81 & 56.75 & 55.51 & 54.55 & 50.18 & 54.28 & 53.61 \\
\hline P. kudriavzevii and SeNPs & 44.34 & 45.67 & 43.76 & 43.32 & 41.62 & 43.54 & 42.43 \\
\hline
\end{tabular}

Effect of P. kudriavzevii and Se-nano formulations on OTA production

For OTA in vitro analyses, all tested $P$. expansum strains were grown in $100 \mathrm{ml}$ of liquid yeast extract sucrose (YES) medium according to Samson et al. (2000). Probiotic yeasts were prepared with approximately $\left(1 \times 10^{7}\right)$ spores suspension and $\mathrm{Se}$-nano-spheres $(3 \mathrm{ml} \mathrm{SeNPs} / 100 \mathrm{ml}$ media) as well as mixture of the former two formulations $(1: 1, \mathrm{v} / \mathrm{v})$ in concentration of $3 \mathrm{ml}$ of the mix/100 ml media and were then inoculated in co-cultures of all mycotoxigenic $P$. expansum strains on the to the YES broth. The $\mathrm{pH}$ of the medium was adjusted to 6.5 with $0.1 \mathrm{~N} \mathrm{NaOH}$ to rule out possible inhibitory effect due to lowered $\mathrm{pH}$ in the growth medium. The flasks were incubated for 14 days at $28^{\circ} \mathrm{C}$ in the dark conditions. Growth of fungal strains and OTA production were estimated. Three replicates for each treatment were used. Three replicates for each treatment were carried out.

\section{Extraction of OTA}

The culture filtrate medium was obtained by filtration through the filter paper (Whatman No. 4). OTA was extracted according to Bragulat et al. (2001) with slight modification. The obtained fungal culture filtrates were individually shaken with chloroform $(1: 1 ; \mathrm{v} / \mathrm{v})$ overnight on an orbital shaker. Then, precipitated by $1.5 \%$ sodium bicarbonate and collected using a generous sized separating funnel by shaking for 30 minutes. The organic upper phase was acidified with $1 \mathrm{~N} \mathrm{HCl}$ and evaporated by rotary evaporation. The residue was air-dried overnight in a chemical fume hood to remove residual solvent. The precipitate was dissolved in $1 \mathrm{ml}$ water: acetonitrile $(3: 1 \mathrm{v} / \mathrm{v})$ and mixed well by vortex for 30 seconds and ready for liquid chromatography.

\section{Qualitative determination of OTA using thin-layer chromatography}

Ten milliliter of samples, with or without treatment, were applied to TLC plates $\left(10 \times 10 \mathrm{~cm}^{2}\right.$, silica gel 60 precoated plates; Merck Darmstadt, Germany) and developed with a 9:1 (v/v) mixture of $\mathrm{CHCl}_{3}$, acetone for $7 \mathrm{~cm}$. OTA analogs were detected as described by Yamazaki et al. (1970).

\section{Quantitative determination of OTA by high-performance liquid chromatography}

The analysis was carried out using HPLC equipped with a binary pump (LC 1110; GBC instrument) C18 column (Hypersil Elite C18; $5 \mu \mathrm{mm}, 250 \times 4.6 \mathrm{~mm}$ ) at the RCMB, AlAzhar University, Cairo, Egypt. An isocratic system with a mobile phase of water-methanol-acetonitrile (55:22.5:22.5, v/v/v) and the flow rate of $1.0 \mathrm{ml} /$ minute were applied. A fluorescence detector
(LC 1255; GBC instrument) with the excitation wavelength set at $365 \mathrm{~nm}$ and the emission wavelength at $440 \mathrm{~nm}$ was used to detect and quantitate the OTA. The injection volume was $20 \mu \mathrm{l}$ for both standard solutions and sample extracts. A standard of OTA (Sigma Chemical Co., USA) was first injected into the HPLC system at different concentrations ranging fom 100 to $1 \mu \mathrm{mg} / \mathrm{ml}$ and compared to samples. OTA concentrations in samples were determined from the standard curve using peak area for quantitation. This methodology was designated by Bragulat et al. (2001).

\section{Transmission electron microscopy examination}

Transmission electron microscopy (TEM) examination was carried out on all samples subjected to the probiotic Pichia kudriavzevii, biosynthesized SeNPs and consolidated plans of both and prepared according to Helmy and Mekawey (2014). Controls and treated cells were fixed in $4 \%$ glutaraldehyde (Sigma, Germany) and post-fixed in $1 \%$ osmium tetroxide (Sigma, Germany) and they were afterward cleaned by $0.1 \mathrm{M}$ sodium cacodylate buffer, $\mathrm{pH}$ 7.4 (Sigma, Germany). After getting rid of the residual osmium tetraoxide, all samples were dehydrated in a graduated acetone (Merck, Germany) series (35\%-100\%). Then, the fixed cells were embedded in Epon and the small blocks of samples were cut with an ultramicrotome (Leica Ultracut-S). The ultra-thin sections were then analyzed at $80 \mathrm{KV}$ using (JEOL 1010) TEM.

\section{Statistical analysis}

All data were statistically analyzed using the General Linear Model procedure of the SPSS var.18. The significance of the differences among treatment groups was determined by Waller-Duncan $k$-ratio. All statements of significance were based on the probability of $p<0.05$ as per Härdle and Simar (2007).

\section{RESULTS AND DISCUSSION}

It has been assessed that $25 \%$ of the world's harvest assembly is spoiled with mycotoxins. OT primarily affect the kidney of all creatures and at high concentration can affect the liver. Their toxic effect has been attributed to inhibition of phenylalanine tRNA synthetase. It is also an immune suppressor, teratogen, and a carcinogen (Kuiper-Goodman and Scott, $1989)$ and its role in the Balkan endemic nephropathy has been implicated. Studies have shown the formation of OTA-DNA adducts in the kidney and bladder tissues of Bulgarian patients undergoing surgery for cancer (Pfohl-Leszkowicz et al., 1993). Several methods were reported to detoxify and/or reduce the OTA; some are using radiation of several doses on the fungal producers. Unfortunately, it was reported that irradiated fungal inocula may produce increased levels of mycotoxins especially aflatox MRSA ins and OT (Applegate and Chipley, 1976; Paster et al., 1985). 
Thus, novel attempts should be given a trial to achieve that purpose. Applying natural materials for such a goal is essential. Probiotic yeast and biogenic SeNPs as a sole and blend strategy were tested in this study for such objective as the first record. They proved to eliminate the OTA production with a very satisfying percentage $(76.08 \%)$ with the $P$. expansum 5 strain as demonstrated by the Table 2. Also, the radial growth of the OTA-producer $P$. expansum strains was greatly affected expressly $P$. expansum5 strain as evidenced by the TEM ultrastructure stability against such probiotic-Nano formulation (Figs. 3 and 4).

In that manner, Aniket et al. (2018) revealed that fussy supplementation of domesticated animals encourages altered by basic selenium in the regular and inorganic frame structures has confirmed to be productive to improve AflaToxin B1 (AFB1) hurtfulness and lethality. Furthermore, Gil et al. (2018) presumed that Pichia pastoris $X-33$ when created in floods of the rice parboiling fabricating amended with glycerol has enhanced the probiotic properties for poultry welfare.

In completion of Agostinho et al. (2016) study, they recommended that probiotics, inorganic selenium (S), and seleniumenhanced probiotics (SP) may encourage mitigating metabolic disorder enhanced by dietary selenium-improved probiotics supplementation than sodium selenite and probiotics gathering. Among the gathering results, including SP has a most extreme impact in enhancing the development execution, biochemical indices, lipid digestion, antioxidative status, histopathological sores, and related quality articulation gene expression in mice sustained a high-fat eating regimen altogether of 80 female albino mice. Yet, there is no confirmation of utilizing probiotic yeasts, especially Pichia kudriavzevii and biogenic SeNPs as a sole and mix methodology in written works till this minute.

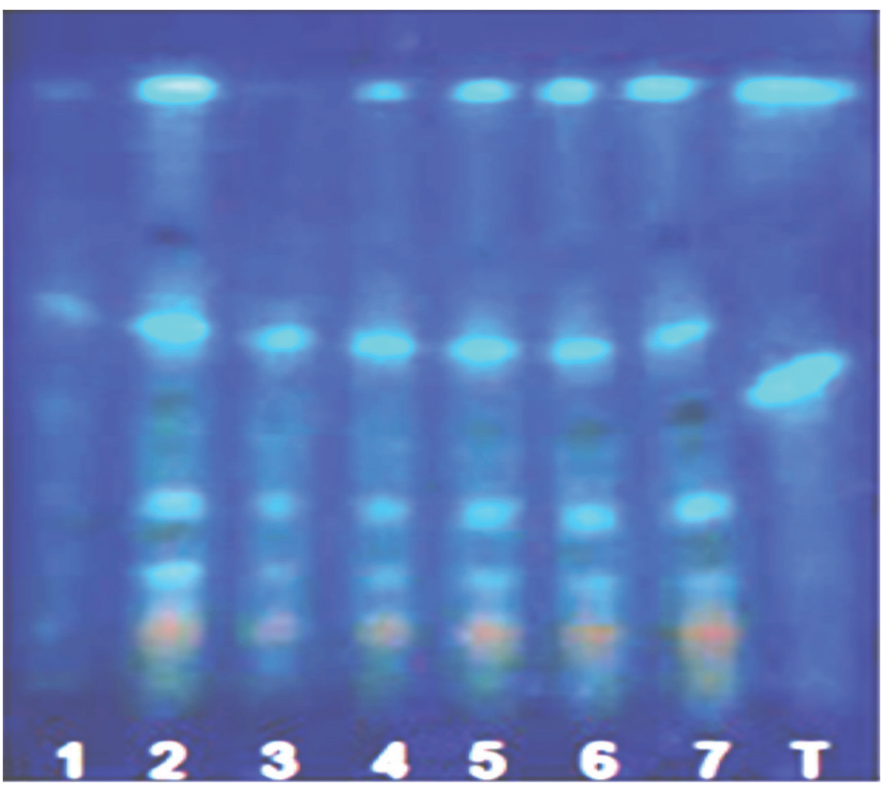

Figure 2. Qualitative chromatographic determination of OTA productivity using TLC technique. Lane 1: Penicllium expansum 1; lane 2: Penicllium expansum2; lane 3: Penicllium expansum3; lane 4: Penicllium expansum4; lane 5: Penicllium expansum5; lane 6: Penicllium expansum6; lane 7: Penicllium expansum 7; and lane T: OTA standard.
Recently, Huo et al. (2014) used a gold NP enhanced surface plasmon resonance imaging chip designed to detect multiple mycotoxins using a competitive immunoassay. They have reported high specific and sensitive detection of aflatoxin B1, OTA, and ZEN with low detection limits of 8, 30, and $15 \mathrm{pg} / \mathrm{ml}$, respectively. No single evidence of using such Se-nanomaterials in a reduction or detoxifying OTA.

\section{Inhibitory effect of probiotic $P$. kudriavzevii and SeNPs formulation against growth of $P$. expansum strains}

Data obtained from a previous survey by Helmy et al. The qualitative assessment fallouts revealed expansum was after treatment with SeNPs at the concentration of $3 \mathrm{ml}$ of SeNPs/100 $\mathrm{ml}$ water cell-free extract.

Concerning the creation of OTA, seven Penicillium expansum strains provided by the $\mathrm{CCU}$ of the RCMB, Al-Azhar University, inspected in this examination using TLC in Figure 2 were recorded surprisingly for the first time. They were subjected to the beforehand announced probiotic Pichia kudriavzevii, biosynthesized SeNPs, and consolidated plans of both to look at their capability to dispose of, detoxificate, and additionally lessening of this mycotoxin. The qualitative assessment fallouts revealed the proficiency of the probiotic Pichia kudriavzevii, biosynthesized SeNPs, and consolidated plans of both against the growth of all the seven explored $P$. expansum strains to produce OTA as compared with the OTA standard. To be confirmed for accurate levels of OTA production by these fungal strains, a quantitative estimation using HPLC was carried out as per Table 2 and Figure 3.
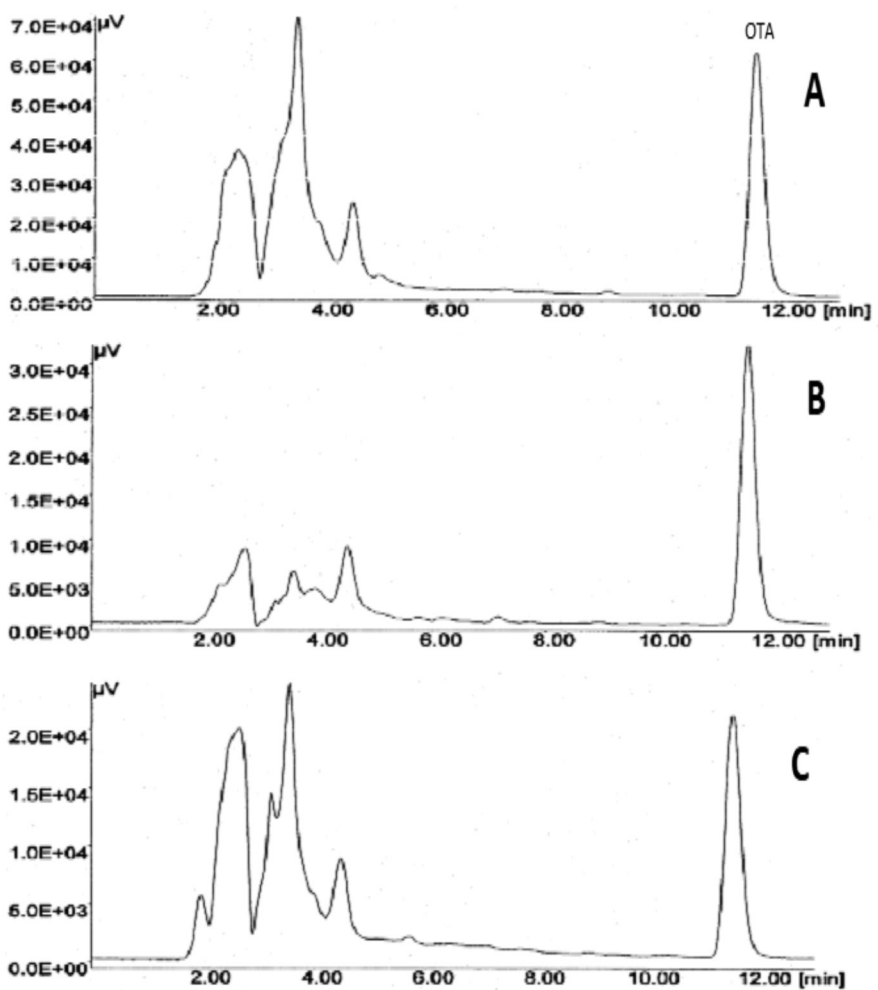

Figure 3. HPLC chromatogram demonstrating quantitative assessment of OTA Production by $P$. expansum5 treated with (A) $P$. kudriavzevii alone; (B) SeNPs alone and (C) the mixture of P. kudriavzevii and biosynthesized SeNPs formulation. 


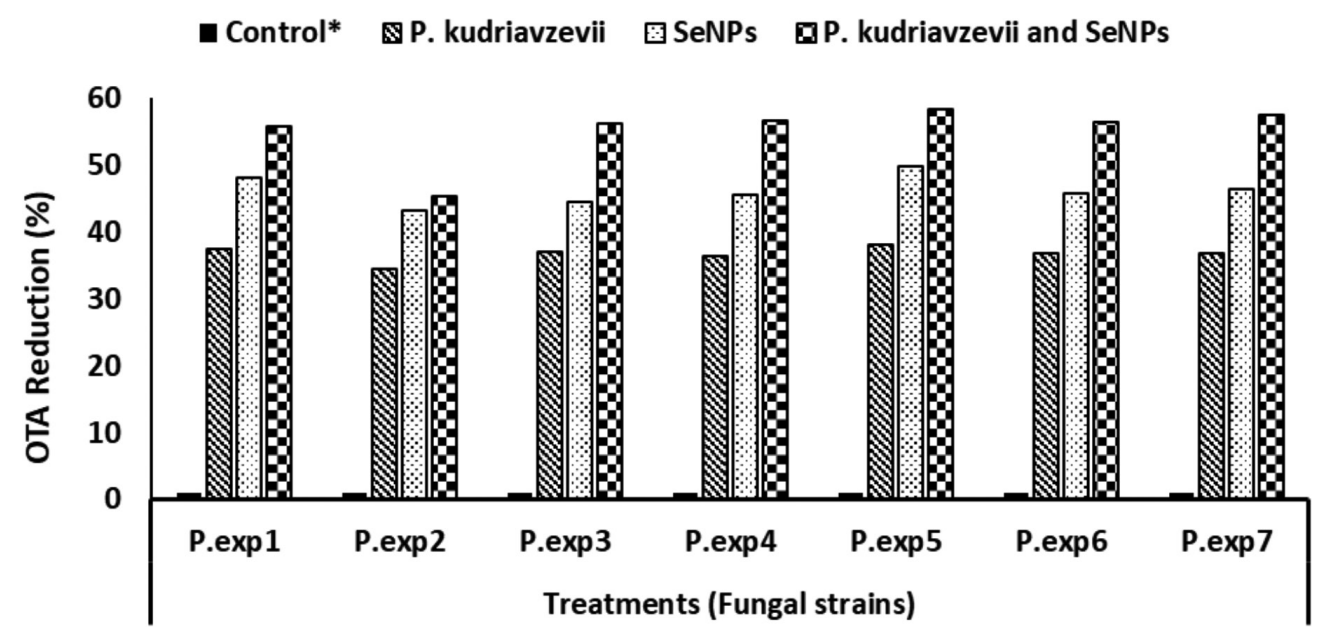

Figure 4. Determination of OTA Reduction (\%) produced by P. expansum strains treated with $P$. kudriavzevii and SeNPs formulations, where control is without any treatment of OTA.

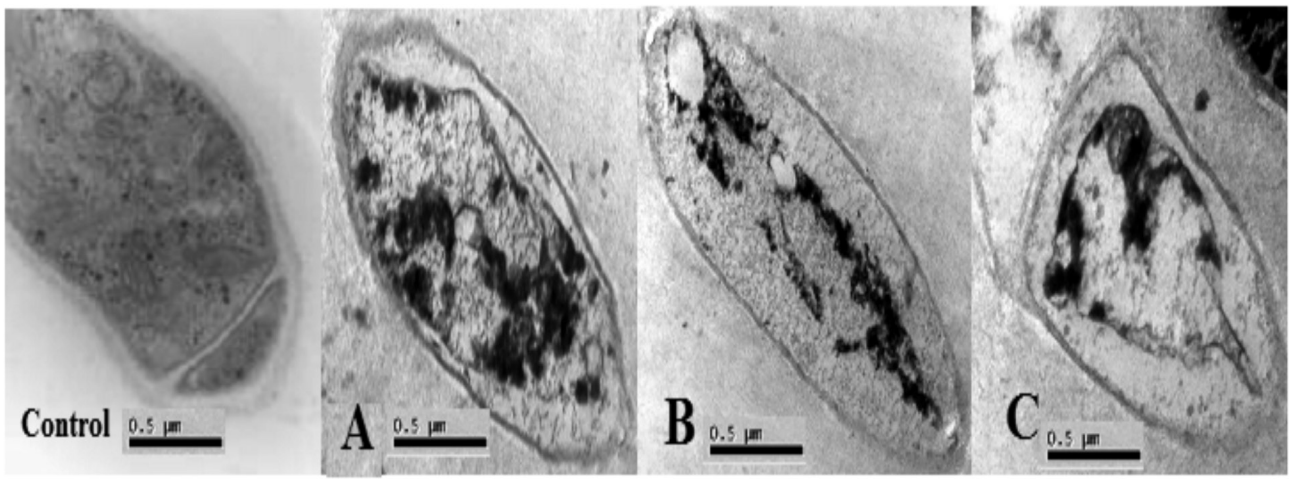

Figure 5. Longitudinal Sectioning of ultrastructure analysis of Penicillium expansum 5 cells treated with (A) SeNPs, (B) probiotic yeast Pichia kudriavzevii, and (C) P. kudriavzevii and SeNPs formulation, where control without any treatment of OTA $(X=20,000)$.

\section{Evaluation of $P$. kudriavzevii and SeNPs formulation on production of OTA}

Data presented in Table 2 represents the OTA reduction percentage (\%) in YES media treated with probiotic Pichia kudriavzevii, biosynthesized SeNPs, and merged plans of both to look at their proficiency to dispose of, detoxificate, and additionally declining of this mycotoxin. Expelling or diminishing of OTA as confirmed by the quantitative assessment using HPLC were observed on every single attempted trial (Figs. 3 and 4). Where the blend of Pichia kudriavzevii and biosynthesized-Selenium nano-plans demonstrated the most OTA evacuation rate created by $P$. expansum 5 by a rate of $(58.38 \%)$. Though the least OTA removal rate was delivered by $P$. expansum 3 by $(34.47 \%)$ treated with SeNPs as it were. The productivity percentage (\%) of OTA were presented in Table 2 but the reduction rate (\%) was illustrated in Figure 4 and calculated according to the following formula as per Ammar and Helmy (2015) utilizing statistical analysis as per Härdle and Simar (2007):

\section{1 - OTA test sample/OTA control $\times 100 \%$}

Concerning the antifungal activity of SeNPs-probiotic formulation against mycotoxigenic $P$. expansum, it has been observed that the growth inhibition level of these strains increased via applying the combination together of SeNPs-probiotic at all different conditions rather than each parameter alone. Although most of the previous studies have evaluated the antibacterial activity of NPs, the antifungal activity of metal NPs against filamentous fungi was still limited. Appropriately, as the discoveries of Parsameher et al. (2017) who indicated, biogenic SeNPs orchestrated with Bacillus sp. had a fitting antifungal activity against fluconazole-resistant and -defenseless Candida albicans strains since these NPs diminished the expression of CDR1 and ERG11 genes related with azole protection. Comparable results were investigated by El-Desouky and Ammar (2016) where the antibacterial and antifungal activity of honey derived NPs were found to have the ability to reduce the mycelial growth and the production of aflatoxins and OTA by Aspergillus flavus and Aspergillus ochraceous, respectively. Recently, both antimicrobial as well as antifungal activities of AgNPs were reported by Mekawey and Helmy (2017), where the AgNPs showed a potent activity against several pathogenic bacterial strains as methicillinresistant Staphylococcus aureus (MRSA) and clinical yeasts as Candida albicans as well as some filamentous mycotoxigenic fungal strains of Aspergillus niger and Penicillium marnefii. 
Regarding the ultrastructural investigations of all fungal strains, especially $P$. expansum 5 strain cells completed with TEM demonstrated the continuous harm of the fungal cells constituents as it appeared from the longitudinal sectioning investigations (Fig. 5). The most elevated impacts were seen among the cytoplasmic martials of $P$. expansum 5 cells treated with the previous blend of Pichia kudriavzevii and biogenic-Selenium nano-combinations that were totally contracted and pulled back far from the cell membrane leaving a gigantic separation region of no organelles. Remarkable cytoplasmic components deformations of $P$. expansum cells with the appearance of some vacuolations as a survival or adaptation mode of the fungal cells were additionally recorded. A noteworthy shrinkage at all researched strains of $P$. expansum cell organelles treated with the previous blend was seen also by the TEM demonstration, for example, cytoplasmic materials, nucleus, nucleolus and mitochondria, the later distorted likewise fit as a fiddle to end up plainly round as opposed to an ordinary oval one of control. Comparable observations were explained by Kim et al. (2007) since the inhibitory effect of AgNPs they investigated on fungal growth may be due to alteration of permeability of cell membrane, release of lipopolysaccharides and membrane proteins, generation of free radicals responsible for the damage of membrane, and dissipation of the proton motive force resulting in the collapse of the membrane potential.

\section{CONCLUSION}

Revolutionize of the antifungal/antimycotoxin potential techniques for utilization in nourishment and encourage protection holds the key to taking care of by shoppers to lessen the employment of innovative additives and added substances in foods and feeds. Thus, the generation of novel safe yet gently handled edibles with the expanded time span of usability is of the general demand. The current study illustrated different strategies of using probiotic and biogenic nanoformulations exhibited antifungal and antimycotoxigenic activities against seven strains of mycotoxigenic P. expansum growth performance and their OTA production. The inhibitory activity was demonstrated by several factors including the ultrastructural deformation of $P$. expansum cells flashed by TEM visualizations and the mycotoxin OTA dimension confirmed by the quantitative assay of HPLC. TEM envisions proved the harm of the fungal cells constituents as it appeared among the distortion of cytoplasmic organelles of $P$. expansum 5 cells treated with the mix of $P$. kudriavzevii and SeNPs with the emergence of some vacuolations as an adaptation survival mode and/or stress-tolerance of the fungal cells. Conclusively, this is a prime statement conveyed OTA diminution using an innovative combination strategy of probiotic yeasts and biogenic-SeNPs which endorsing the impact of utilizing such novel natural mixture for the detoxification and elimination of OTA production. This natural invention could be recommended to utilize as a food/feed additive, during storage and as a prophylactic agent in pre- and post-harvest crops. This investigation recommends an extensive in situ research studies to prove the application of such unusual innovation on the detoxification/reduction of higher levels of OTA in food and feedstuffs as well as in pre- and post-harvest crops in a large scale.

\section{CONFLICTS OF INTEREST}

There is no conflict of interest and no affiliations with or involvement in any organization or entity with any financial interest.

\section{ACKNOWLEDGMENT}

Author would like to express the deepest gratitude to professor Dr. Khalil A. A., for his meticulous, sincere and patient guidance throughout the writing steps of this work.

\section{REFERENCES}

Adegoke GO, Letuma P. Strategies for the prevention and reduction of Mycotoxins in developing countries. In: Makun HA (ed.). Book chapter of mycotoxin and food safety in developing countriesMycotoxicology. InTech Janeza Trdine 9, Rijeka, Croatia, pp 123-37, 2013.

Ammar HAM, Helmy EA. Ecophysiological conditions affecting growth and ochratoxin A production by novel Aspergillus terreusHA2 and Aspergillus fumigatus HAl isolated from Egyptian rice grains. Egypt J Bot, 2015; 12(2):11-22.

Aniket L, Roch-Chui Y, Cheng-Chun C, Je-Ruei L, Kuan-Chen C. Protective and detoxifying effects conferred by dietary selenium and curcumin against AFB1-mediated toxicity in livestock: a review. Toxins, 2018; 10(25):1-18.

Association of Official Analytical Chemists (AOAC). Official methods of analysis 18th AOAC, Method 971.22. Published Chemists, Washington, DC, 2005.

Applegate KL, Chipley JR. Production of ochratoxin A by Aspergillus ochraceous NRRL-3174 before and after exposure with 60Co irradiation. Appl Environ Microbiol, 1976; 31:349-53.

Asmathunisha N, Kathiresan KA. Review on biosynthesis of nanoparticles by marine organisms. Colloid Surf B Biointerfaces, 2013; 103:283-7.

Bragulat MR, Abarca ML, Cabanes FJ. An easy screening method for fungi producing OTA in pure cultures. Int J Food Microbiol, 2001; 71:139-44.

Dalie D, Pinson-Gadais L, Atanasova-Penichon V, Marchegay G, Barreau C, Deschamps A, Richard-Forget F. Impact of Pediococcus pentosaceus strain L006 and its metabolites on fumonisin biosynthesis by Fusarium verticillioides. Food Control, 2012; 23(2):405-11.

Dorner JW, Cole RJ, Connick WJ, Daigle DJ, McGuire MR, Shasha BS. Evaluation of biological control formulations to reduce aflatoxin contamination in peanuts. Biocontrol, 2003; 26:318-24.

El-Desouky TA, Ammar HAM. Honey mediated silver nanoparticles and their inhibitory effect on aflatoxins and ochratoxin A. J Appl Pharm Sci, 2016; 6(06):83-90.

Fink-Gremmels J, Malekinejad H. Clinical effects and biochemical mechanisms associated with exposure to the mycoestrogen zearalenone. Ani Feed Sci Technol, 2007; 137:326-41.

Gabriel O. Adegoke and Puleng Letuma. (2013). Strategies for the prevention and reduction of Mycotoxins in developing countries. Book chapter of mycotoxin and food safety in developing countries Mycotoxicology, Edited by Hussaini Anthony Makun. Published by InTech Janeza Trdine 9, 51000 Rijeka, Croatia. 123-137.

Gil de los Santos D, Gil de los Santos JR, Gil-Turnes C, Gaboardi G, Fernandes Silva L, FrancËa R. Probiotic effect of pastoris X-33 produced in parboiled rice effluent and YPD medium on broiler chickens. PLoS ONE, 2018; 13(2):e0192904.

Härdle W, Simar L. Applied multivariate statistical analysis. 2nd edition, Springer, Heidelberg, p 420, 2007.

Helmy EA. Nano-biotechnology breakthrough and food-packing industry. Microbial Biosystems, 2016; 1(1):50-69.

Helmy EA, Abdul Aziz SS. In vitro isolation and evaluation of probiotic attributes of three dairy yeast isolates against clinical and foodborne pathogens, 2018 (Unpublished Data).

Helmy EA, Mekawey AA. Envision of the microbial contact with mycosynthesized silver nanoparticles. Res J Pharm Biol Chem Sci, 2014; 5(5):344-54.

Helmy EA, Shehata RM, Abdul Aziz SS. Ecofriendly green biogenic synthesis of selenium nano spheres by Trichoderma harzianum, 2018 (Unpublished Data). 
Huo S, Jin S, Ma X, Xue X, Yang K, Kumar A, Wang PC, Zhang $\mathrm{J}, \mathrm{Hu} \mathrm{Z}$, Liang XJ. Ultrasmall gold nanoparticles as carriers for nucleusbased gene therapy due to size-dependent nuclear entry. ACS Nano J, 2014; 8(6):5852-62.

Khalil AA, Abou-Gabal AE, Elfaramawy AM, Khaled AE, Abdellatef AA. Lactic acid bacteria as antimycotic and antimycotoxins agents against Toxigenic Fusarium species associated to maize grains stored in Egyptian markets. J Pure Appl Microbiol, 2013; 7(Spl. Edn.):93-105.

Kim JS, Kuk E, Yu KN, Kim JH, Park SJ. Antimicrobial effects of silver nanoparticles. Nanomed Nanotechnol Biol Med, 2007; 3:95-101.

Koteswara RV, Aruna B, Girisham S, Madhusudhan RS. Effect of indigenous fungi on ochratoxin A produced by two species of Penicillium. Ani Nutr, 2016; 2:225-8.

Kuiper-Goodman T, Scott PM. Risk assessment of the mycotoxin ochratoxin A. Biomed Environ Sci, 1989; 2:179-248.

Leonardo P, Corbo MR, Milena S, Antonio B. Ochratoxin A removal by yeasts after exposure to simulated human gastrointestinal conditions. J Food Sci, 2016; 81(11):12-24.

Mahoney M, Molyneux RJ, Kim JH, Campbell BC, Waiss AC, Hagerman AE. Aflatoxigenesis induced in Aspergillus flavus by oxidative stress and reduction by phenolic antioxidants from tree nuts. World Mycotoxin J, 2010; 3(1):49-57.

McCormick S. Microbial detoxification of mycotoxins. J Chem Ecol, 2013; 39:907-18.

Mekawey AI, Helmy EA. Elucidative physiological optimization of silver nanospheres biogenesis by molds. Int J Nanotechnol Allied Sci, 2017; 1(1):30-44.

Nido SA, Shituleni SA, Mengistu BM, Liu Y, Khan AZ, Gan F, Kumbhar S, Huang K. Effects of selenium-enriched probiotics on lipid metabolism, antioxidative status, histopathological lesions, and related gene expression in mice fed a high-fat diet. Biol Trace Elem Res, 2016; 171(2):399-409.

O'Brien E, Dietrich DR. Ochratoxin A: the continuing enigma. Crit Rev Toxicol, 2005; 35:33-60.

Parsameher N, Rezaei S, Khodavasiy S, Salari S, Hadizade S, Kord M, Ayatollahi Mousavi SA. Effect of biogenic selenium nanoparticles on ERG11 and CDR1 gene expression in both fluconazole-resistant and -susceptible Candida albicans isolates. Curr Med Mycol, 2017; 3(3):16-20.
Paster N, Barkai-Golan R, Padova R. Effect of gamma radiation on ochratoxin production by the fungus Aspergillus ochraceus. J Sci Food Agric, 1985; 36:445-9.

Pfohl-Leszkowicz A, Grosse Y, Castegnaro M, Nicolov IG, Chernozemsky IN, Bartsch H, Betbeder AM, Creppy EE, Dirheimer G. Ochratoxin A-related DNA adducts in urinary tract tumors of Bulgarian subjects. IARC Sci Publ, 1993; 124:141-8.

Qi L, Li Y, Luo X, Wang R, Zheng R, Wang L, Li Y, Yang D, Fang W, Chen Z. Detoxification of zearalenone and ochratoxin A by ozone and quality evaluation of ozonized corn. J Food Addit Contam 2016; 33(HYPERLINK "https://www.tandfonline.com/toc/tfac20/33/11" 11):12-24.

Saeger SD, Kris A, Siska C. Report from the 5th International Symposium on Mycotoxins and Toxigenic Moulds: Challenges and Perspectives (MYTOX) Held in Ghent, Belgium, May 2016. Toxins 2016; 8:146-83.

Samson RA, Hoekstra ES, Frisvad JC, Filtenborg O. Introduction to food- and airborne fungi. Centraalbureau voor Schimmelcultures-Utrecht, Utrecht, The Netherlands, 389 p, 2000.

Stoimenov PK, Klinger RL, Marchin GL, Klabunde KJ. Metal oxide nanoparticles as bactericidal agents. Langmuir, 2002; 18:6679-86.

Thalita C, Fernández-Cruzb ML, Verdec SC, Armando V, Luís A. Gamma irradiation effects on ochratoxin A: Degradation, cytotoxicity and application in food. Food Chem, 2018; 240:463-71.

Yamazaki M, Maebayashi Y, Miyaki K. Production of Ochratoxin A by Aspergillus ochraceous isolated in Japan from moldy rice. Appl Microbiol, 1970; 20: 452-4.

Yehia RS, Ahmed OS. In vitro study of the antifungal efficacy of zinc oxide nanoparticles against Fusarium oxysporum and Penicillium expansum. Afr J Microbiol Res, 2013; 7(19):1917-23.

How to cite this article:

Helmy EAM. Detoxification and anti-mycotoxigenic effect of Ochratoxin A produced by Penicillium expansum species utilizing probiotic Pichia kudriavzevii and biogenic-selenium nano-formulations. J Appl Pharm Sci, 2019; 9(S1): 001-008. 